\title{
Isolation of chemical compounds from Sorbus tianschanica Rupr by high-speed counter-current chromatography
}

\author{
Changzhi $\mathrm{Yu}^{1}$, Yonggang Zheng ${ }^{1}$, Le $\mathrm{Li}^{1}$, Guojun Wang ${ }^{1}$, Yingchun $\mathrm{Li}^{1}$, Tao $\mathrm{Wu}^{2}$ and Hui Tang ${ }^{1 *}$ \\ ${ }^{1}$ Pharmacy School of Shihezi University, Shihezi, Xinjiang, 832000, China. \\ ${ }^{2}$ Xinjiang Technical Institute of Physics and Chemistry, Chinese Academy of Science, 830011 Urumqi, China.
}

Accepted 19 June, 2012

\begin{abstract}
Sorbus tianschanica Rupr has been used as a pharmacologically valuable plant in Xinjiang to treat tuberculosis, asthma, cough and gastritis for a long time. Four compounds including three flavonoids and chlorogenic acid were isolated and purified by high-speed counter-current chromatography (HSCCC) from S. tianschanica Rupr. The two-phase solvent system composed of $n$-hexane - ethyl acetate - n-butanol - water (2:6:1:8, v/v). Consequently, chlorogenic acid (1), Quercetin-3-O-(6"-Omalonyl)- $\beta$ - $D$-glucoside (2), Kaempferol-3-O-(6"-O-malonyl)- $\beta$ - $D$-glucopyranoside (3) and Kaempferol-3$O-\beta$-D-glucopyranoside (4) were obtained from $250 \mathrm{mg}$ crude extracts with purities of 93, 91, 89 and $96 \%$, respectively in a single run. The chemical structures were confirmed by mass spectrometer (MS), ${ }^{1} \mathrm{H}$ nuclear magnetic resonance (NMR) and ${ }^{13} \mathrm{C}$ NMR. To our knowledge, compounds (2) and (3) were obtained from $S$. tianschanica Rupr for the first time.
\end{abstract}

Key words: High-speed counter-current chromatography, Sorbus tianschanica Rupr, flavonoids, chlorogenic acid.

\section{INTRODUCTION}

Sorbus tianschanica Rupr, a member of family Rosaceae, is distributed in mountainous regions of Central Asia and west of China. It has been widely used as a pharmacologically valuable plant in Xinjiang to treat tuberculosis, asthma, cough and gastritis (Zhang et al., 1973). Pharmacological research indicates that crude extracts of flavonoids from $S$. tianschanica exhibits a wide range of biological activities (Yu et al., 2004), such as against myocardial ischemia reperfusion injury in rats (Fu et al., 2010), anti-myocardial ischemia (Zhang et al., 2009) and antitussive. It is reported that major constituents in $S$. tianschanica are phenolic acids, aglycones, flavonoids, which include quercetin, tutin, hyperin and flavonols (Yu et al., 2004; Li et al., 2010). However, few new constituents in this plant have been

\footnotetext{
*Corresponding author. E-mail: yu5406@hotmail.com.
}

described in recent years. Considering such bioactive compounds of flavonoids from $S$. tianschanica, it is prerequisite to develop an efficient method of isolating each compound with high purity for further pharmacological research and quality control.

Although column chromatography and preparative liquid chromatography (LC) are widely used in isolation and purification of flavonoids (Rijke et al., 2006; Salib et al., 2006; Gil-Izquierdo et al., 2001), the methods are time-consuming and sample-losing during the separation and purification processes. Thus, a more efficient strategy is required in order to obtain compounds of low content in original plant with high sample recovery. Highspeed counter-current chromatography (HSCCC), a type of liquid-liquid partition chromatography, has great merits of higher yield and recovery in extracting target compounds than those of conventional column chromatography (Ito, 2005). Separation process of HSCCC is based on the composition of two solvent 
system, providing flexibility in choosing separation solvents for different target compounds. HSCCC has great advantages of $100 \%$ sample recovery, simplicity to scale up and high yield of sample. Therefore, this technique has been extensively applied for separation and purification of natural products (Ito et al., 2011; Lee et al., 2011; Shi et al., 2011; Hu et al., 2008), especially for flavonoids (Liu et al., 2005; Yang et al., 2009; Wu et al., 2009; Wei et al., 2011).

The study has succeeded in developing an efficient approach for isolation and purification of four bioactive compounds from $S$. tianschanica by HSCCC in one single run. Their chemical structures have been confirmed by high performance liquid chromatographymass spectrometer (HPLC-MS), ${ }^{1} \mathrm{H}$ nuclear magnetic resonance (NMR) and ${ }^{13} \mathrm{C} N M R$. Up to now, there are no previous scientific literature reports of using HSCCC for isolation and purification flavonoids from S. tianschanica. As far as we know, two compounds obtained in this research, quercetin-3-O-(6"-O-malonyl)- $\beta$-D-glucoside and kaempferol-3-O-(6"-O-malonyl)- $\beta$-D-glucopyranoside are for the first time extracted from the plant- $S$. tianschanica.

\section{MATERIALS AND METHODS}

The HSCCC instrument was TBE-300B (Tauto Biotechnique Company, Shanghai, China) equipped with a $280 \mathrm{ml}$ coil column (i.d. of the tubing $1.9 \mathrm{~mm}$ ) and a $20 \mathrm{ml}$ sample loop. The revolution speed could be adjusted from 0 to $1000 \mathrm{rpm}$. TBP 5002 constant flow pump (Tauto Biotechnique Company, Shanghai, China), a Model ultraviolet (UV) 20 detector was used to record absorption (Tianzhao, Hangzhou, China).

The HPLC equipment used Agilent 1100 HPLC system including G1311A pump, G1314A detector, Rheodyne 7725i injection valve with a 20- $\mu$ l loop, G1322A degasser and Agilent HPLC workstation.

MS used Applied Biosystem API-4000 (ABi, USA). NMR spectrometer used Mercury plus 400 NMR and 600 NMR (Varian Inc., USA).

Acetonitrile applied for HPLC were chromatographic grade (Fisher Scientific, USA), and water was distilled water. All organic solvents were analytical grade (GuangFu, Tianjin, China). AB-8 macroporous resin (Nankai University, Tianjin, China) were employed for crude extracts preparation.

\section{Preparation of crude extracts}

ST leaves $(1 \mathrm{~kg})$ were extracted twice with $60 \%$ ethanol by ultrasonic means. The extracts were mixed together and concentrated to remove ethanol. After filtrated with cotton, the filtrate was loaded onto AB-8 macroporous resin, and then washed successively with water and $60 \%$ ethanol, respectively. The eluant of $60 \%$ ethanol was concentrated by using a rotary evaporator and then a freeze-dryer to give dried crude powder (74 g).

\section{Selection of two-phase solvent system}

The two-phase solvent system was selected according to the partition coefficient $(K)$ of each target component. Acceptable $K$ of the target compounds should be in the range 0.2 to 5.0 (Ito, 2005).
The $K$-values were determined by HPLC as follows: Two-phase solvent system was prepared by mixing various solvent thoroughly in a separatory funnel and left for a period at room temperature. Then equal volume of the upper and the lower phase were taken out and mixed again, afterwards, $120 \mathrm{mg}$ crude extracts were added into the two-phase system. Then both the upper and the lower phase solution were detected by HPLC. The obtained peak area of the upper phase $\left(A_{1}\right)$ and the lower phase $\left(A_{2}\right)$. K-values according to the equation: $K=A_{1} / A_{2}$.

\section{Preparation of two-phase solvent system and sample solution}

The two-phase solvent system composed of n-hexane - ethyl acetate - n-butanol - water $(2: 6: 1: 8, v / v)$ was used for HSCCC separation. When all the solvent mixed thoroughly, the two-phases were separated and degassed for 30 min before using.

The sample solution for HSCCC separation was prepared by dissolving $250 \mathrm{mg}$ crude extracts in $20 \mathrm{ml}$ lower phase solvent.

\section{HSCCC separation procedure}

The upper phase was used as the stationary phase; the lower phase was used as the mobile phase. Firstly, the column was fully filled with the upper phase, and then the HSCCC rotated at 800 rpm, while the lower phase was pumped into the column at a flow rate of $2.0 \mathrm{ml} \mathrm{min}^{-1}$. When the hydrodynamic equilibrium, which can be directly recognized by observing the record from the workstation, was established in the column, $20 \mathrm{ml}$ of the sample solution containing $250 \mathrm{mg}$ of the crude extract was injected into the column. The temperature was controlled at $25^{\circ} \mathrm{C}$. The effluent was continuously monitored with a UV detector at $254 \mathrm{~nm}$. Peak fractions were collected according to the UV chromatogram.

\section{HPLC analysis and identification of HSCCC peak fractions}

The crude extracts and each peak fraction were obtained by HSCCC were analyzed by HPLC with a Waters $C_{18}$ column (150 $\mathrm{mm} \times 4.6 \mathrm{~mm}$ i.d. $5 \mu \mathrm{m})$. The mobile phase was acetonitrile $(A)$ and $0.4 \%$ phosphoric acid solution $(B)$ in gradient mode as follows: 0 to $10 \mathrm{~min}, 15$ to $22 \%(\mathrm{~A}), 10$ to $20 \mathrm{~min}, 22$ to $30 \%(A)$, flow rate $1.0 \mathrm{ml}$ $\mathrm{min}^{-1}$. The effluent was monitored at $254 \mathrm{~nm}, 25^{\circ} \mathrm{C}$. Purities of each peak fraction were given by HPLC. The chromatograms of crude extracts and peak fractions separated by HSCCC are shown in Figure 1.

\section{RESULTS AND DISCUSSION}

\section{Optimization of two-phase solvent system and other conditions of HSCCC}

The two-phase solvent system was selected by the $K$ values of each target compound. Table 1 shows the $K$ values, which were also determined by HPLC or tested in HSCCC. The results indicated that when chloroform methanol - water $(4: 3: 2, \mathrm{v} / \mathrm{v})$ was used as the two-phase solvent, $K$-values of each target compound were too low to suit each compound to be separated. The system consisting of ethyl acetate - n-butanol - water (3:1:3, v/v) (Zhou et al., 2005) was then tested and the results displayed $K$-values were too high for each compound. 

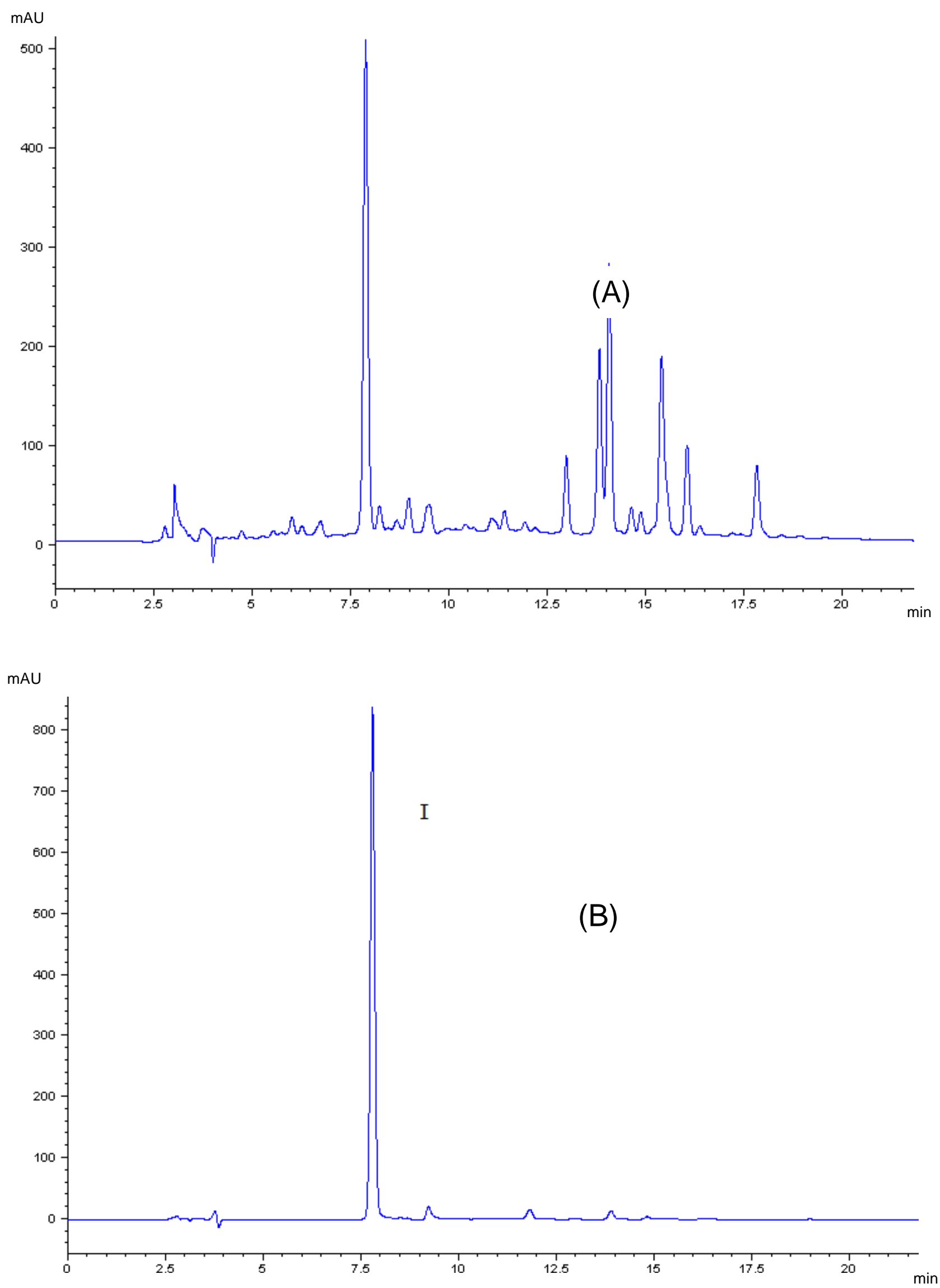
Yu et al. $\quad 5145$
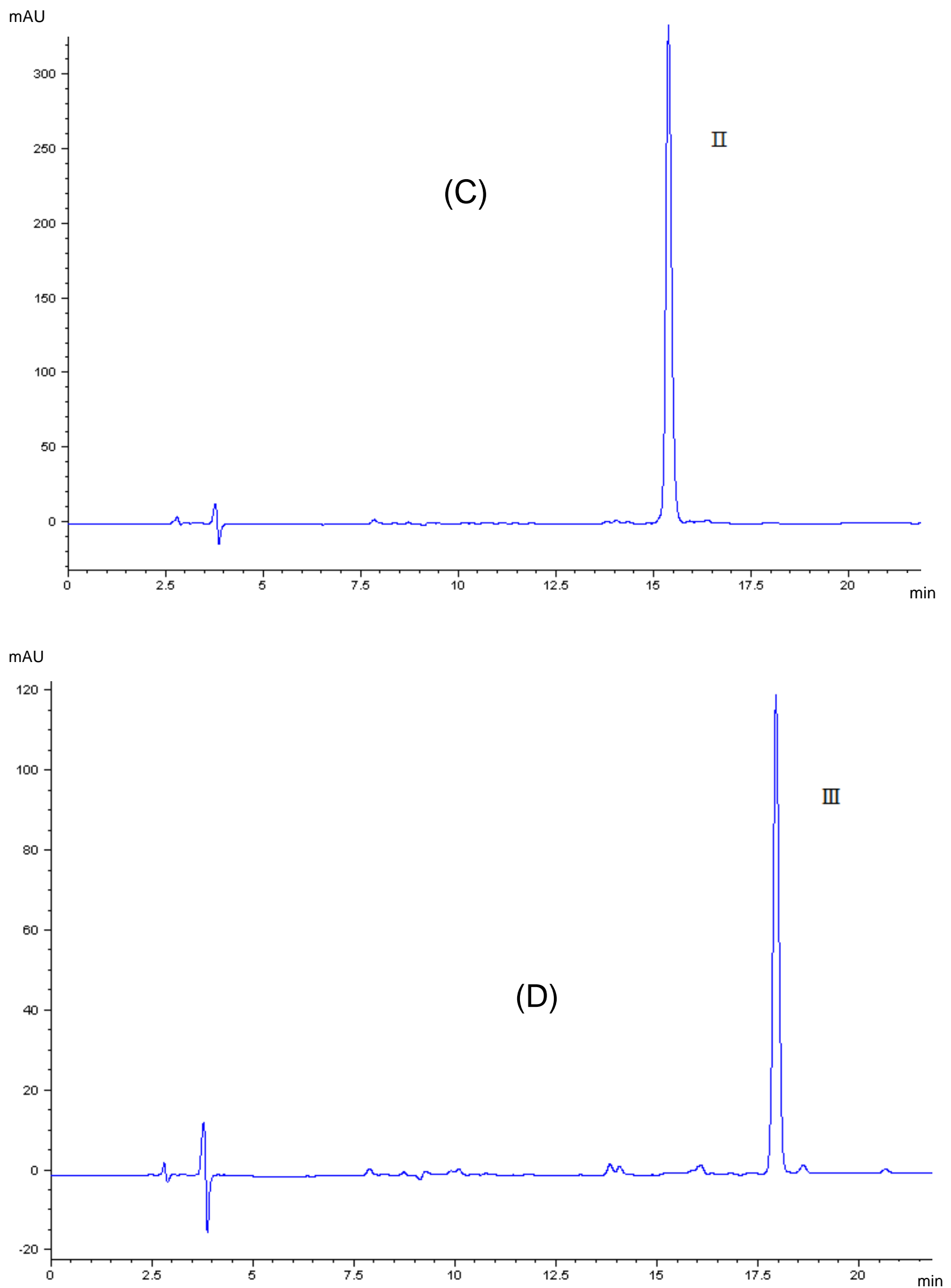


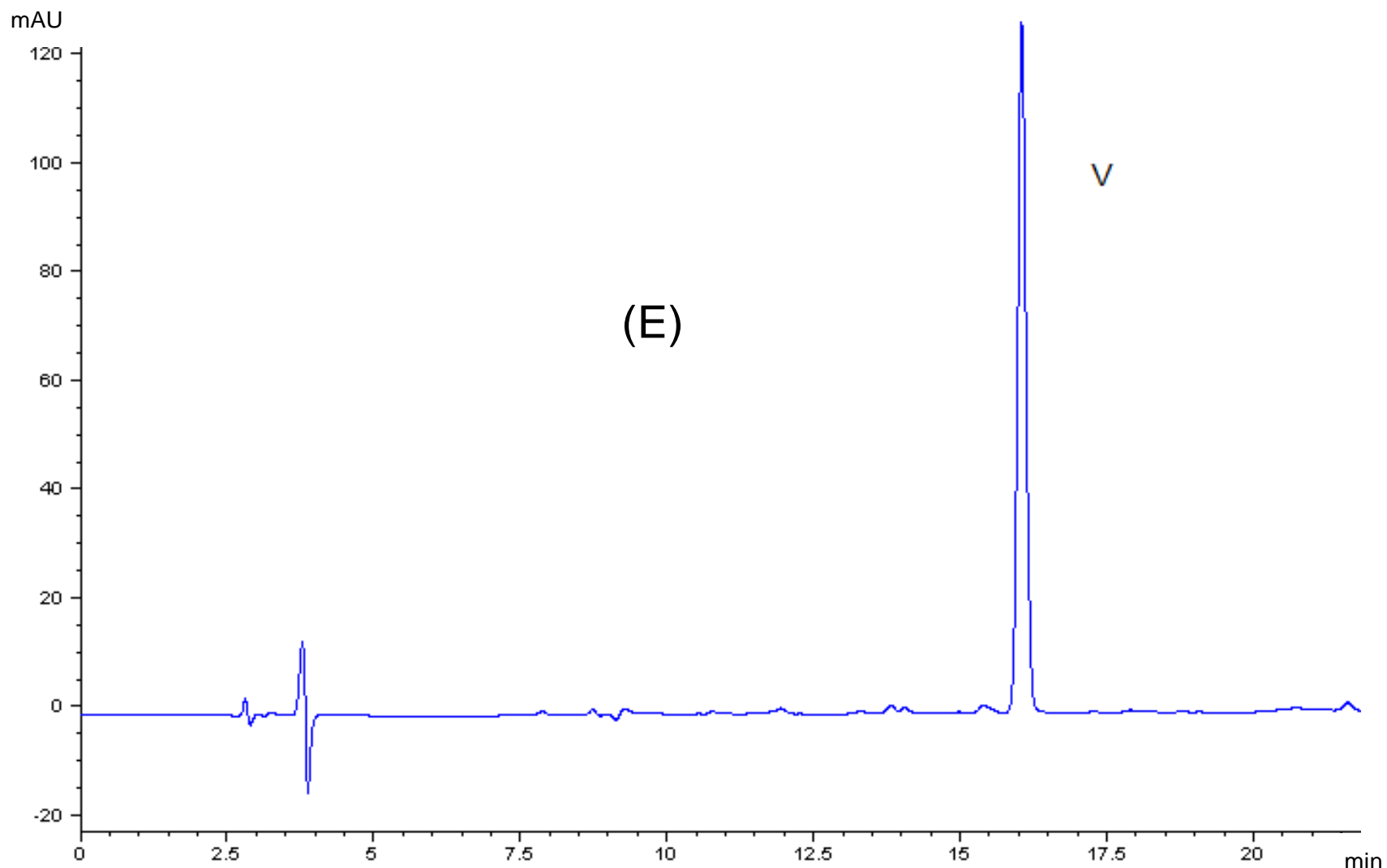

Figure 1. HPLC chromatogram of crude extract and HSCCC peak fractions. (A), Crude extract from S. tianschanica Rupr; (B-E), HPLC analyses of the purified HSCCC peak fractions I-III and V.

Table 1. The $K$-values of target components measured in several solvent systems.

\begin{tabular}{lcccc}
\hline \multirow{2}{*}{ Solvent system (v/v) } & \multicolumn{3}{c}{$\boldsymbol{K}$} \\
\cline { 2 - 5 } & $\mathbf{( 1 )}$ & $\mathbf{( 2 )}$ & $\mathbf{( 3 )}$ & $\mathbf{( 4 )}$ \\
\hline Chloroform - methanol - water $(4: 3: 2, \mathrm{v} / \mathrm{v})$ & $>0.01$ & $>0.01$ & 0.02 & 0.02 \\
Ethyl acetate - n-butanol - water $(3: 1: 3, \mathrm{v} / \mathrm{v})$ & 3.02 & 0.75 & 5.70 & 2.98 \\
Ethyl acetate - n-butanol - water (4:1:6, v/v) & 2.63 & 2.44 & 9.19 & 1.43 \\
Ethyl acetate - n-butanol - water $(6: 1: 8, \mathrm{v} / \mathrm{v})$ & 0.21 & 3.31 & 3.51 & 0.98 \\
n-Hexane - ethyl acetate - n-butanol - water (1:6:1:8, v/v) & 0.18 & 0.46 & 1.70 & 3.01 \\
n-Hexane - ethyl acetate - n-butanol - water (1.5:6:1:8, v/v) & 0.16 & 0.27 & 1.16 & 2.83 \\
n-Hexane - ethyl acetate - n-butanol - water (2:6:1:8, v/v) & 0.16 & 0.22 & 0.85 & 1.65 \\
\hline
\end{tabular}

Thus, it took a great of time for separation, and the retention percentage of the stationary phase was low. Then optimization was done at the volume ratio of $(4: 1: 6$, $\mathrm{v} / \mathrm{v})$, and got better results. However, since the fact that the volume ratio of $n$-butanol was still large resulted in high viscosity of solvent system, the obtained retention percentage of the stationary phase was too low. $K$-values was acceptable with ethyl acetate - n-butanol - water $(6: 1: 8, \mathrm{v} / \mathrm{v})$ as solvent system, but two compounds of the extract cannot be fully separated, giving purities both lower than $65 \%$. When n-hexane - ethyl acetate - nbutanol - water (1:6:1:8, v/v) and (1.5:6:1:8, v/v) was used, separation of four compounds can be realized but required quite a long time. When $n$-hexane - ethyl acetate - n-butanol - water $(2: 6: 1: 8, \mathrm{v} / \mathrm{v})$ was applied, the four compounds were well separated, and the time was acceptable. The purities of each target compound were higher than $89 \%$. Thereby, n-hexane - ethyl acetate - nbutanol - water (2:6:1:8, v/v) was used as the two-phase solvent system of HSCCC in the following study. 


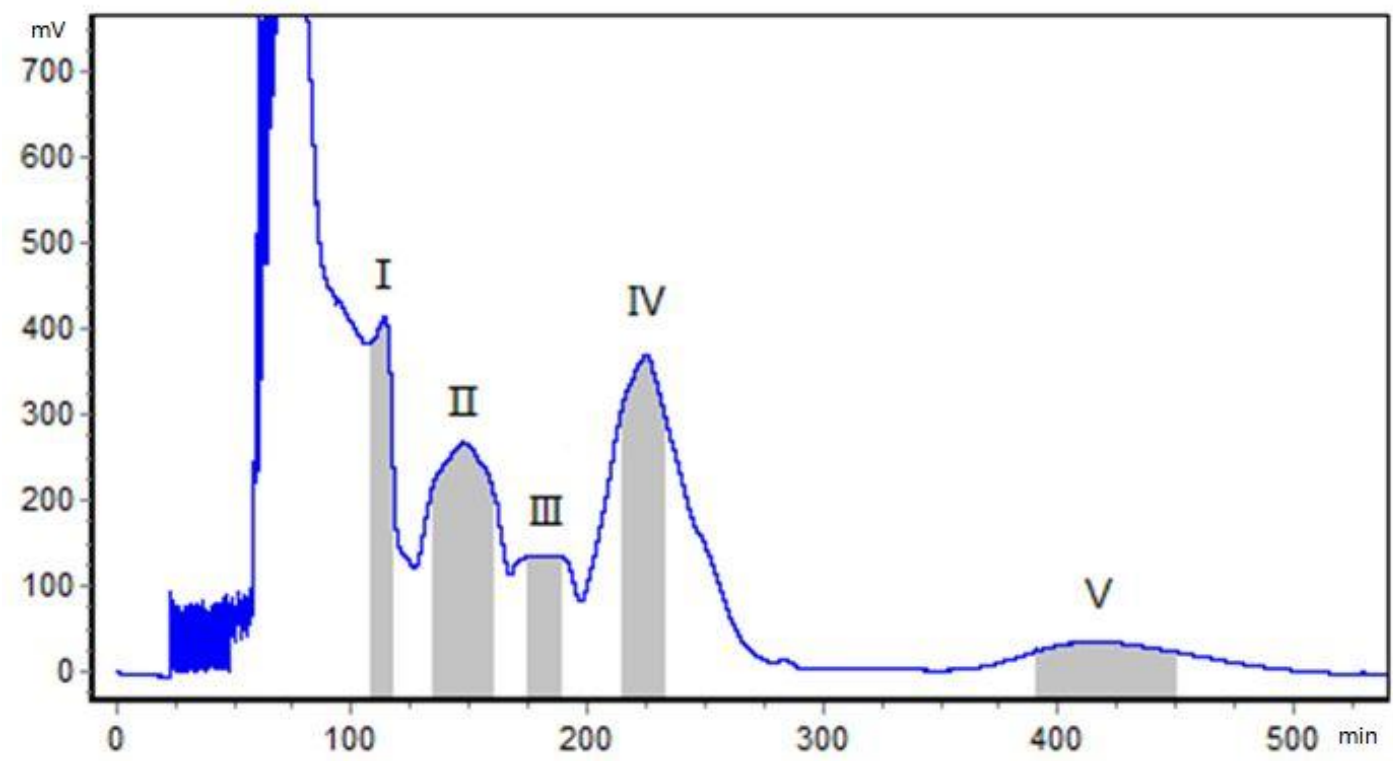

Figure 2. HSCCC chromatogram of crude extract from $S$. tianschanica Rupr. Concentration of HSCCC, tow-phase solvent system: n-hexane - ethyl acetate - n-butanol - water (2:6:1:8, v/v), flow rate, 2.0 $\mathrm{ml} / \mathrm{min}$; revolution speed, $800 \mathrm{rpm}$; detection wavelength, $254 \mathrm{~nm} ; 250 \mathrm{mg}$ crude sample; separation temperature, $25^{\circ} \mathrm{C}$; retention percentage of the stationary phase, $63.5 \%$.

Other factors, for instance, the flow rate of mobile phase, the temperature and the revolution speed of separation process, were also considered. Increasing flow rate of mobile phase will reduce the time of separation, while it will sacrifice retention percentage of the stationary phase and resolution of each compound. In the study, we used flow rate $2.0 \mathrm{ml} \mathrm{min}^{-1}$, a good resolution can be obtained with retention percentage of the stationary phase $63.5 \%$. For the Peak V, since it is the last one in the chromatogram, the flow rate was increased from 2.0 to $3.5 \mathrm{ml} \mathrm{min}^{-1}$ after $300 \mathrm{~min}$. This compound can be collected with high purity in less time as well. Theoretically, rise in temperature could improve the HSCCC separation, but in practice we found it displayed poor effect, so $25^{\circ} \mathrm{C}$ was selected for separation. The experiment was performed at the revolution speed of $800 \mathrm{rpm}$.

The HSCCC chromatogram is shown in Figure 2. Peak I, compound (1), collected 110 to $115 \mathrm{~min}, 27.2 \mathrm{mg}$; Peak II, compound (2), collected 135 to $160 \mathrm{~min}, 17.0 \mathrm{mg}$; Peak III, compound (3), collected 170 to $190 \mathrm{~min}, 12.2$ mg; Peak IV, compounds (4), we obtained two compounds mixed, ESI-MS/MS shown the two compounds have the same molecular weight and aglycon; Peak V, compound (5), collected 390 to $450 \mathrm{~min}$, $25.6 \mathrm{mg}$.

\section{Structural identification}

The structure identification of compounds (1), (2), (3) and
(4) were carried out by ESI-MS/MS, ${ }^{1} \mathrm{H} N M R$ and ${ }^{13} \mathrm{C}$ NMR. According to the data given in reference, four compounds were chlorogenic acid, compound (1), (Jung et al., 1999). Quercetin-3-O-(6"-O-malonyl)- $\beta$ - $D$ glucoside, compound (2), (Kamata et al., 2008). Kaempferol-3-O-(6"-O-malonyl)- $\beta$ - $D$-glucopyranoside, compound (3), (Wald et al., 1989). Kaempferol-3-O- $\beta-D$ glucopyranoside, compound (5), (Nguelefack et al., 2011). The structures of compounds are shown in Figure 3.

\section{Conclusion}

HSCCC has been successfully used to preparative isolation of three flavonoids and chlorogenic acid from $S$. tianschanica. About $27.2 \mathrm{mg}$ chlorogenic acid (1), 17.1 mg quercetin-3-O-(6"-O-malonyl)- $\beta$ - $D$-glucoside (2), 12.2 mg Kaempferol-3-O-(6"-O-malonyl)- $\beta$ - $D$-glucopyranoside (3) and $25.6 \mathrm{mg}$ astragalin (4) were obtained from 250 mg crude extracts with purities of $93,91 \%, 89$ and $96 \%$. The compounds (2) and (3) were obtained from $S$. tianschanica for the first time.

By using this method, we can not only get at least four compounds in one time, but obtaining sufficient amounts ready for further scientific study can be also expected, which is hardly to realize with column chromatography and preparative HPLC, wherein repeating work is needed to get the comparable quantity. These compounds will be employed for further pharmacological studies or as reference substances. 

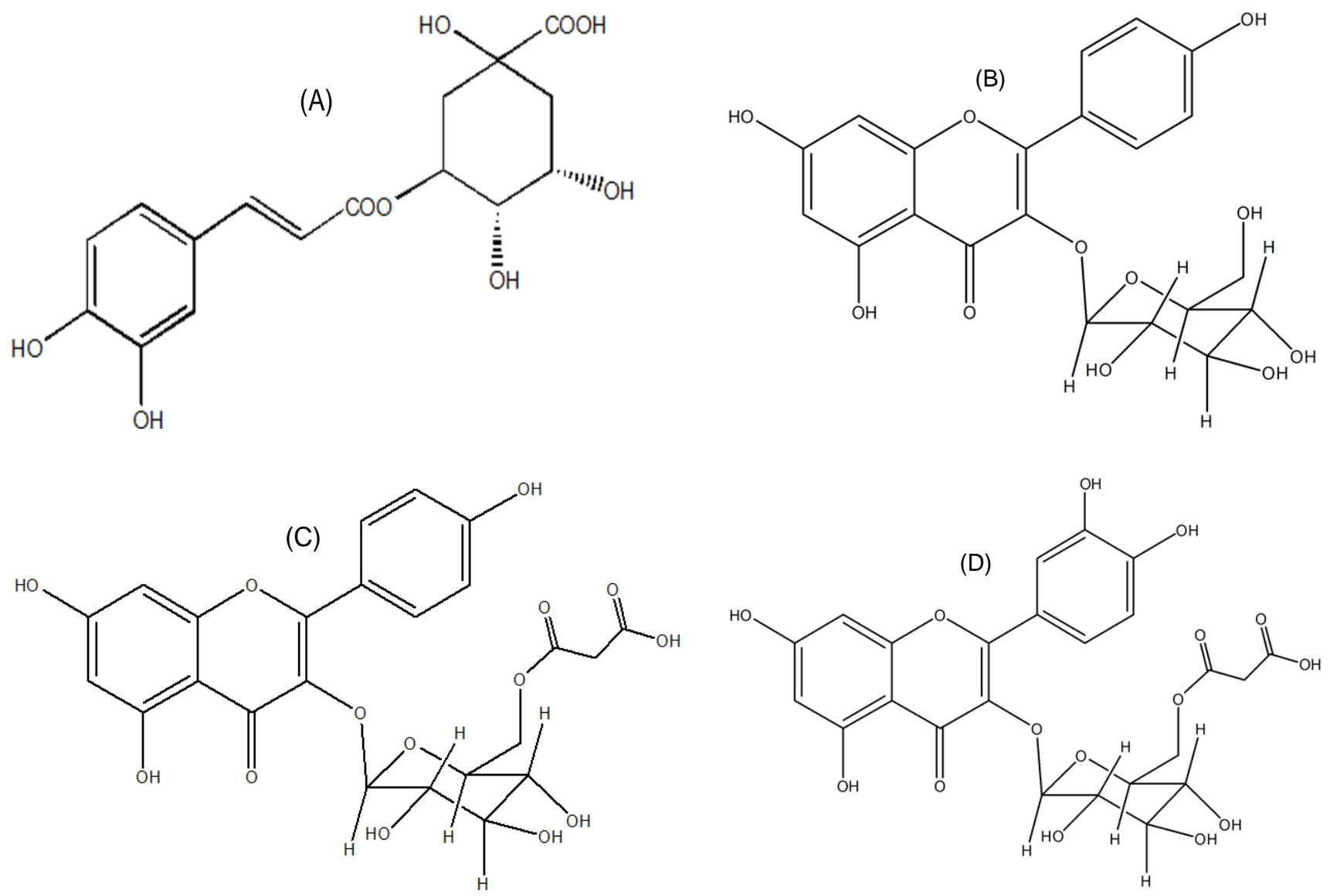

Figure 3. Chemical structures of compounds from S. tianschanica Rupr. (A), Chlorogenic acid; (B), Quercetin-3-O-(6"-Omalonyl)- $\beta$-D-glucoside; (C), Kaempferol-3-O-(6"-O-malonyl)- $\beta$ - $D$-glucopyranoside; (D), Kaempferol-3-O- $\beta$ - $D$-glucopyranoside.

\section{ACKNOWLEDGEMENTS}

The authors is acknowledge the financial support from Natural science foundation of china $(81260627 / \mathrm{H} 2803)$; Medical special Program of Xinjiang production and construction Corps (2006GG52); Technology support Program of Xinjiang production and construction Corps (2008ZJ14).

\section{REFERENCES}

Fu W, Liu T, Yang CY, Wang T, Zheng QS, Tang H, Wang ZH (2010). Protective effect of total flavonoids from Sorbus tianschanica $L$ leaf against myocardial ischemia reperfusion injury in rats. Chinese Pharmacol. Bull. 26:251-254.

Gil-Izquierdo A, Mellenthin A (2001). Identification and quantitation of flavonols in rowanbeer (Sorbus aucuparia L.) juice. Eur. Food Res. Technol. 213:12-17.

Hu DJ, Liu M, Xia X, Chen DJ, Zhao FS, Ge M (2008). Preparative isolation and purification of Altertoxin I from an Alternaria sp. By HSCCC. Chromatographia 67:836-867.

Ito $Y$ (2005). Golden rules and pitfalls in selectiong optimum conditions for high-speed counter-current chromatography. J. Chromatogr. A. 1065:145-168.

Ito $\mathrm{Y}$, Zhang $\mathrm{YM}$, $\mathrm{He} \mathrm{J}$ (2011). Semi-Preparative Isolation and
Purification of Three Tauro-Conjugated Cholic Acids from Pulvis Fellis Suis by HSCCC Coupled with ELSD Detection. Chromatographia 73:361-365.

Jung HA, Park JC, Chung HY, Kim J, Choi JS (1999). Antioxidant Flavonoids and Chlorogenic Acid from the Leaves of Eriobotrya japonica. Arch. Pharmacal. Res. 22:213-218.

Kamata K, Seo S, Nakajima J (2008). Constituents from leaves of Apocynum venetum L. J. Nat Med. 62:160-163.

Lee YS, Kim SH, Kim JK, Lee S, Jung SH, Lim SS (2011). Preparative isolation and prification so seven isoflavones from Belamcanda chinensis. Phytochem. Anal. 22:468-473.

Li L, Tang H, Wu T, Yu CZ (2010). Chemical Composition of Sorbus tianschanica Leaves. Chem. Nat. Compd. 46:811-812.

Liu RM, Li AF, Sun AL, Cui JC, Kong LY (2005). Preparative isolation and purification of three flavonoids from the Chinese medicinal plant epimedium koreamum Nakai by high-speed counter-current chromatography. J. Chromatogr. A. 1064:53-57.

Nguelefack TB, Mbakam FHK, Tapondjou LA, Watcho P, NguelefackMbuyo EP, Ponou BK, Kamanyi A, Park HJ (2011). A Dimeric Triterpenoid Glycoside and Flavonoid Glycosides with Free RadicalScavenging Activity Isolated from Rubus rigidus var. camerunensis, Arch. Pharmacal Res. 34:543-550.

Rijke ED, Out P, Niessen WMA, Ariese F, Gooijer C, Brinkman UAT (2006). Analytical separation and detection methods for flavonoids. J. Chromatogr. A. 1112:31-63.

Salib JY, Michael HN, Ei-Nogoumy SI (2008). New lactoyl glycoside quercetin from melia zaedarach leaves. Chem. Nat. Compd. 44:1315. 
Shi J, Li G, Wang H, Zheng J, Suo Y, Liu Y (2011). One-step Separation of Three Flavonoids from Poacynum hendersonii by High-speed Counter-current Chromatography. Phytochem. Anal. 22:450-454.

Wald B, Wray V, Galensa R, Herrmann K (1989). Malonated flavonol glycosides and 3,5-Dicaffeoylqunicacid from pears. Phytochemistry 28:663-664.

Wei Y, Razwan SM, Sutherland IA, Fisher D (2011). Separation of Delphinidin-3-O-sambubioside, Cyanidin-3-O-sambubioside and $\mathrm{p}$ Coumaric Acid from Cranberry by CCC Followed by prep-HPLC Using Robotic CCC Solvent System Selection. Chromatographia 74:367-373.

Wu T, Lin JB, Yang Y, Abdulla R, Chen J, Akber-Aisa A (2009). H Preparative isolation of three flavonoids from Flos Gossypii by highspeed counter-current chromatography. Sep. Purif. Technol. 66:295298.

Yang Y, Huang Y, Gu DY, Yili A, Sabir G, Aisa HA (2009). Separation and Purification of Three Flavonoids from Helichrysum arenarium (L.) Moench by HSCCC. Chromatographia 69:936-967.
Yu M, Li X (2004). Review on research of the chemical constituents and pharmacological activities of Sorbus Tianschanica L. J. Liaoning Coll. TCM. 6:364-366.

Zhang Q, Li G, Tang H, Rui M, Li L (2009). Screening for anti-myocardial ischemia fractions from extract of Sorbus Tianschanica L. Chinese Pharmacol. Bull. 25:277-278.

Zhang YQ (1973). Xinjiang Herbal Committee, Flora Xinjiangensis, Xinjiang People's Publishing House, Urumqi. p. 62.

Zhou X, Peng JY, Fang GR, Wu YT (2005). Isolation and purification of flavonoid glycosides from Trollius ledebouri using high-speed counter-current chromatography by stepwise increasing the flow-rate of the mobile phase. J. Chromatogr. A. 1092:216-221. 